\title{
Hemisection of a severely decayed mandibular molar: a case report
}

\author{
Cendranata Wibawa Ongkowijoyo, Latief Mooduto, Deavita Dinari and Riski Setyo Avianti \\ Department of Conservative Dentistry, \\ Faculty of Dental Medicine, Universitas Airlangga, \\ Surabaya, Indonesia
}

\begin{abstract}
Background: Dental implants gained popularity as the treatment to replace grossly decayed teeth. However, with the increasing evidence and the difficulty to manage complications associated with dental implants, clinicians are pushed to opt for a more conservative approach. Case: A male patient with a chief complaint of frequent food impaction in a severely decayed mandibular molar wished to retain his tooth. Upon thorough examination, the carious lesion extended to furcal area that rendered the distal root unsalvageable. However the mesial root can be retained; thus, hemisection was proposed. Case Management: Root canal treatment was carried out in the mesial canals. Then, the tooth was split mesio-distally. The distal root was extracted, and the mesial root was retained. Subsequently, the tooth was restored with PFM crown. Conclusion: Hemisection with subsequent prosthetic rehabilitation can be a viable alternative to retain severely decayed mandibular molar.
\end{abstract}

Keywords: Hemisection; root resection; compromised tooth

Correspondence: Latief Mooduto, Department of Conservative Dentistry, Faculty of Dental Medicine, Universitas Airlangga. Jl. Mayjend. Prof. Dr. Moestopo No. 47 Surabaya 60132, Indonesia. E-mail: latiefmdt@yahoo.co.id

\section{INTRODUCTION}

The decision to extract or to retain grossly decayed tooth is not always a straightforward task. In the past decades, compromised teeth were more likely to be extracted and replaced with dental implants; thus, dental implants gains more popularity among dentists. ${ }^{1-3}$

However, due to increasing evidence of dental implant biological and technical complications and the difficulty to manage those complications, clincians are pushed to opt for a more conservative approach, which is to postpone extraction and to retain teeth. ${ }^{1,4,5}$ In endodontics discipline, a compromised tooth can be retained with various endodontic surgery approaches. For example, with apex resection, bicuspidization, surgical extrusion, and hemisection. ${ }^{6}$ In this case report, the management of a severely decayed mandibular molar up to furcation level with hemisection and prosthetic restoration will be presented.

\section{CASE REPORT}

A 45-year-old male patient came to the Conservative Dentistry Department, Dental Hospital of Universitas Airlangga with a chief complaint of a frequent food impaction in the region of left mandibular molar. The tooth was treated previously but remain unfinished. The patient reported no pain associated with the offending tooth. General medical history was non-contributory.

Upon clinical examination, there was no extraoral and intraoral tissue abnormality. The offending tooth number 36 presented with an extensive carious lesion distally, with a subgingival margin, and a little remaining of temporary filling material. No pain reported after percussion and bite test.

Radiographic examination revealed a large distal carious lesion extending to furcal area of 36 . There was no periapical lesion associated with both mesial and distal roots. According to AAE classification, 36 was diagnosed as "previously treated tooth".

According to clinical and radiographic examination, the distal root of 36 was unsalvageable. However, the mesial root along the mesial part of crown was intact. The patient wished to retain the tooth. Therefore, root canal treatment of mesial canals, hemisection and extraction of distal roots, followed by porsthetic restoration with porcelain fused to metal (PFM) crown was proposed. The patient agreed with the proposed treatment plan.

\section{CASE MANAGEMENT}

$1^{\text {st }}$ visit - endodontic treatment: Tooth 36 was isolated with rubber dam. Conservative access opening was performed to preserve sound tooth structure. 2 orifices (MB and ML) 
was found in the mesial root. Negotiation was performed with D-finder 10 (Mani, Japan), apical patency and glide path were achieved with the same instrument. Working length was determined with electronic apex locator and was confirmed with periapical radiograph $(20 \mathrm{~mm})$.

The canals were flooded with $\mathrm{NaOCl} 2.5 \%$. Protaper Next X1 and X2 (Denstply, USA) were used to shape the canals with crown down technique. Root canal cleaning was done with $30 \mathrm{G}$ side vented irrigation needle (OneMed, Indonesia) with the following protocol: EDTA $17 \%$ for 1 minute, flushed with sterile aquadest; and $\mathrm{NaOCl} 2.5 \%$ + activation with Endoactivator (Dentsply, USA) for 30 seconds, repeated 3 times, flushed with sterile aquadest.

The canals were subsequently dried with endo-suction and obturated with Protaper Next X2 gutta percha (Dentsply, USA) and AH-Plus sealer (Dentsply, USA). Resin composite (Z 350 XT, 3M, Germany) was used as coronal sealing material.

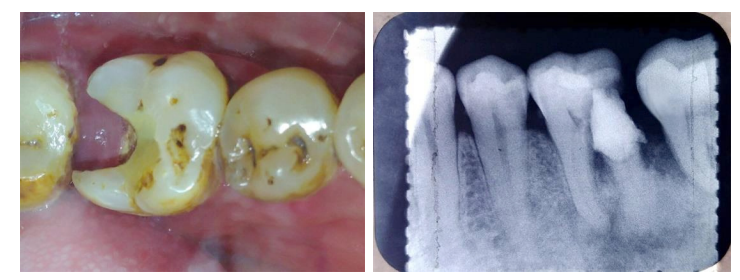

Figure 1. Pre-operative clinical \& radioraph.
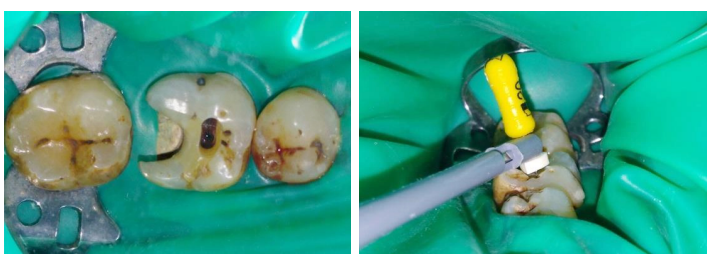

Figure 2. Isolation, access opening, and working length determination.
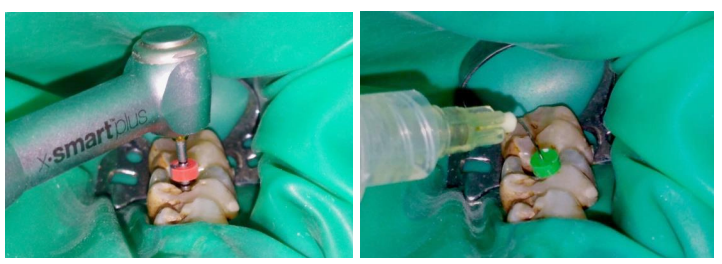

Figure 3. Shaping and cleaning. $2^{\text {nd }}$ visit - hemisection: Prior to surgery, extraoral and intraoral soft tissue were rubbed with chlorhexidine $2 \%$ antiseptic solution. Mandibular block anesthesia was done with lidocaine $+1: 80.000$ adrenaline (Pehacaine, Phapros, Indonesia).

Before hemisection, the separation site was determined in mesio-distal and apico-coronal aspect to achieve a precise separation. With long thin coarse diamond bur, the distal and mesial roots of 36 was separated right at the furcation level.

Surgical elevator was used to engage between the roots and ascertain separation is complete ${ }^{7}$. Then, the distal root was elevated and extracted.

Furcal roof was subsequently probed and smoothened with fine diamond bur to eliminate any roughness present. The extraction socket was then irrigated with saline to remove debris. ${ }^{5,8}$ Carbonate apatite block (Gamacha, Indonesia) was applied as bone graft material and covered with native collagen membrane (Botiss, Germany).
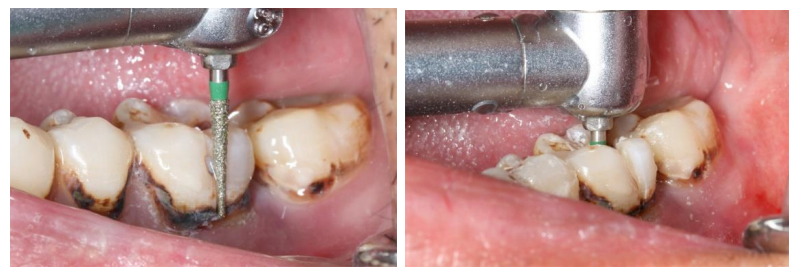

Figure 5. Separation of mesial \& distal roots.
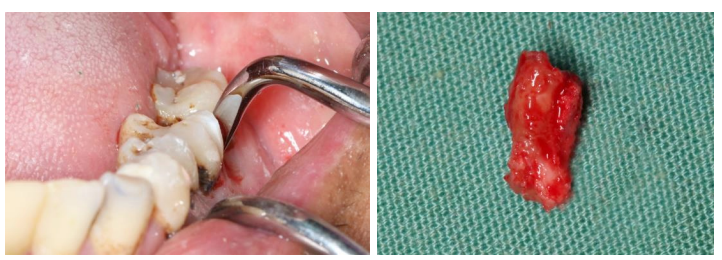

Figure 6. Extraction of distal root.
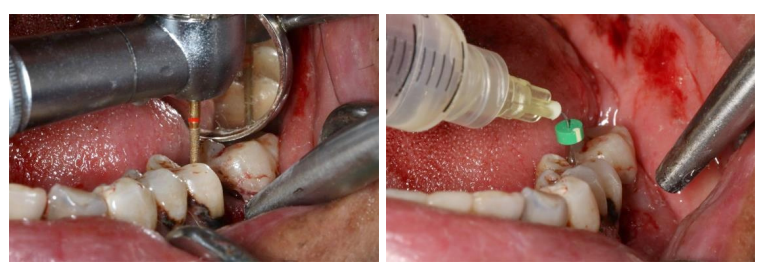

Figure 7. Smoothening and irrigation.
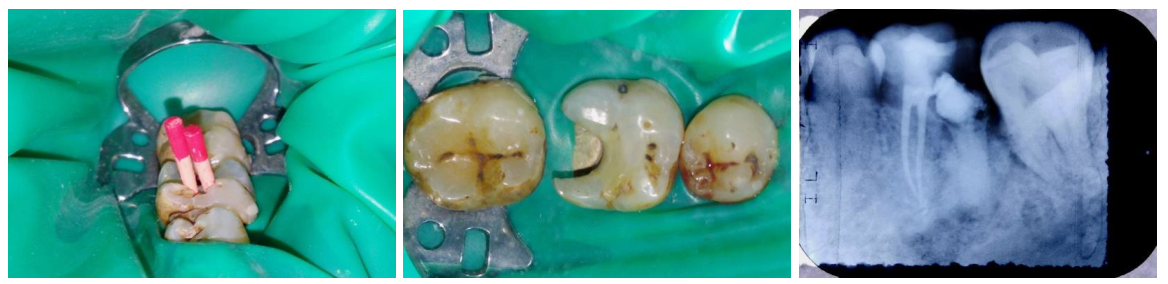

Figure 4. Obturation and coronal sealing.

https://e-journal.unair.ac.id/CDJ 

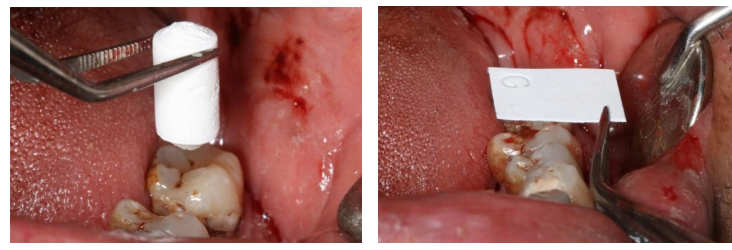

Figure 8. Bone graft and membrane.

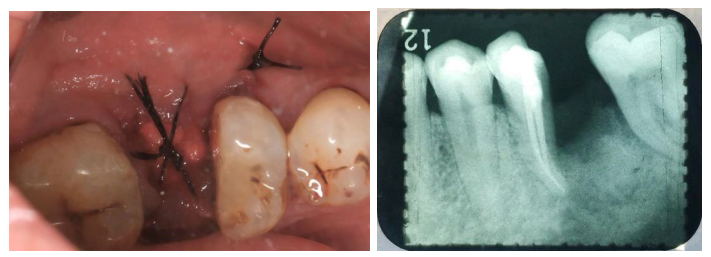

Figure 9. Suturing and periapical radiograph.

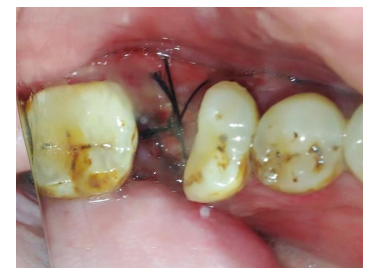

Figure 10. Suture removal.

Gingiva was sutured to secure bone graft and membrane in place. Periapical radiograph was taken to evaluate the hemisection procedure. The patient was given prescription of antibiotics and antiinflammatory medications as well as post-hemsiection instructions.

$3^{\text {rd }}$ visit - suture removal: 1 week after hemisection, the soft tissue healed unevently. Sutures were removed. $4^{\text {th }}$ visit - full coverage crown preparation: 3 months after hemisection. The soft tissue and bone healed uneventfully. The remaining mesial part of tooth 36 was prepared for full coverage PFM crown. Mesial marginal ridge of 37 was also prepred for mesial rest, providing additional support for the PFM crown.

Impression for working cast was made with PVS in double-step technique. Prepared tooth 36 was subsequently temporised. Lab instructions was then sent for PFM crown fabrication with 3M 3 shade.

$5^{\text {th }}$ visit - PFM crown try in \& cementation: Temporary crown was removed and PFM crown was tried and evaluated for its marginal fit, proximal fit, and occlusion. Isolation was carried out using split dam technique. Tooth 36 and 37 was then cleaned prior to cementation. Subsequently, PFM crown was cemented with RMGIC cement.

\section{DISCUSSION}

According to American Association of Endodontics, hemisection is defined as: "The surgical separation of a

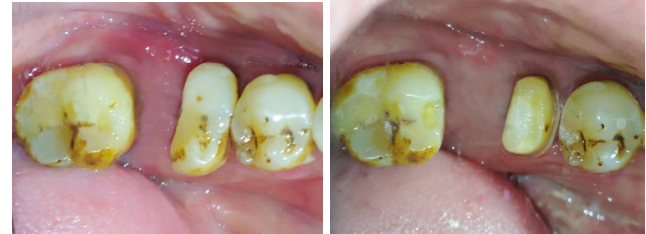

Figure 11. Full coverage crown preparation.

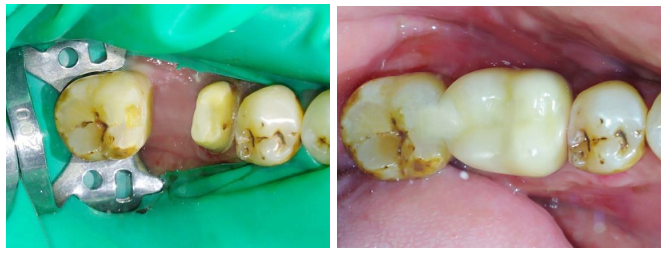

Figure 12. Isolation \& PFM crown insertion.

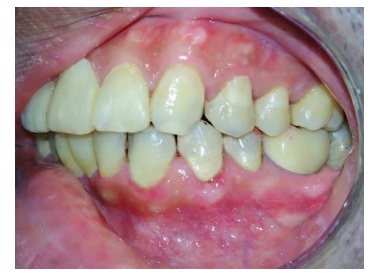

Figure 13. After PFM crown cementation.

multirooted tooth, usually a mandibular molar, through the furcation in such a way that a root and the associated portion of the crown may be removed or retained."

Hemisection is one of the arsenals within endodontics discipline which utilizes both conservative and surgery measures to retain compromised teeth. Indications and contraindications of hemisection are: $:^{5,7,8,10-13}$ Patient wishes to retain the tooth; Multirooted teeth in which one or more of the roots can be retained due to sound hard and periodontal tissue, but the other cannot be retained due to; Extensively decayed; Fractured; Severely resorbed; Large iatrogenic perforation; Severe root proximity with adjacent tooth inadequate for a proper embrassure space; Involved with an extensive periodontal disease which compromises the periodontal support; and Symptomatic and persistent periapical lesion which cannot be endodontically treated due to blockage, ledges, or presence of separated instruments.

Hemisection is a very valuable treatment modality where a part of multirooted tooth acting as a terminal abutment of a short-span bridge fails..$^{8,14,15}$ Contraindications: ${ }^{5,7,8,10-13}$ Patient does not wish to retain the tooth; Single rooted teeth; Multirooted teeth in which the roots are fused; Multirooted teeth in which the furcation is located far apical from the alveolar crest (taurodontism); The root to be retained cannot be endodontically treated due to blockage, ledge, or presence separated instrument; The root to be retained does not have adequate sound tooth structure due to excessive endodontic instrumentation previously; Inadequate periodontal support surrounding the root to be retained; Unfavorable crown:root 
ratio within the root to be retained; Multirooted teeth located outside proper arch form; and other considerations which necessitate complete extraction of the tooth.

Regardless being frequently performed to replace compromised teeth, dental implants has several additional risks and drawbacks. Following tooth extraction, the extraction socket must be grafted. Otherwise, tissue resorption will occur, compromising the volume for implant placement and will present additional challenge due to proximity to vital anatomic structure. Unlike teeth, peri-implant soft and hard tissue needs to be meticulously managed to prevent failure. Moreover, due to the absence of periodontal ligament as a shock absorber, dental implant hardware complications are not rare, such as screw loosening, abutment fracture, and veneering porcelain chipping. In terms of financial perspective, dental implants might incur more cost to patients compared to a more conservative measures. ${ }^{1,4}$

Hemisection, enabling the clinician to preserve to retain part of a compromised tooth, offers several advantages. Surrounding hard and soft tissue health can be more easily maintained with proper marginal fit, occlusion and oral hygine. Also, periodontal ligament which act as shock absorber and proprioception is maintained. ${ }^{6,12,14}$ Hemisection also offers psychological advantages over extraction because a part of tooth is still retained..$^{10}$ In terms of financial perspective, hemisection might incur less cost to patients compared to dental implants. ${ }^{1,4,6,11}$

Hemisection has a very high survival rate. ${ }^{3,13}$ According to a systematic review evaluating more than hemisected 90 teeth with up to 40 years follow-up, it was found that hemisection has a survival rate of $95 \% .{ }^{4}$ In order to achieve such a high survival rate, several measures are needed to be strictly followed, such as: $:^{1,5,7,11-13,15}$ Case selection: tooth to be retained has a large, long, divergent root and crown; the root to be retained does not act as a terminal abutment of a long-span fixed dental prosthesis; Endodontic treatment: access should be as small as possible; lateral condensation obturation technique must be avoided; Surgery: atraumatic extraction; removing roughness on furcal roof, eliminating root concavity for easy oral hygiene measures; socket preservation of the extraction socker; Restoration: limiting the use of endodontic post; making cuspal inclines less steep to reduce lateral forces; sanitary pontic.

\section{CONCLUSION}

With careful case selection, hemisection with subsequent rehabilitation with prosthetic crowns can be a viable treatment modality to save an extensively decayed mandibular molar.

\section{REFERENCES}

1. Mokbel N, Kassir AR, Naaman N, Megarbane J. Root Resection and Hemisection Revisited . Part I : A Systematic Review. 2019;39(1):11-32.

2. Kurian B, Swapna D V, Nadig RR, Vedawathi B. Hemisection-Survival of fittest. Int J Appl Dent Sci [Internet]. 2016;2(2):71-3. Available from: http://www.oraljournal. com/pdf/2016/vol2issue2/PartB/2-2-22.pdf

3. Sharma S, Sharma R, Ahad A, Gupta ND, Mishra SK. Hemisection as a Conservative Management of Grossly Carious Permanent Mandibular First Molar. J Nat Sci Biol Med. 2018;9(1):97-9.

4. Megarbane J-M, Kassir A, Mokbel N, Naaman N. Root Resection and Hemisection Revisited. Part II: A Retrospective Analysis of 195 Treated Patients with Up to 40 Years of Follow-up. Int J Periodontics Restorative Dent. 2018;38(6):783-9.

5. Singh M, Satish C. Hemisection in an Era of Implants. Open Access J Dent Sci. 2018;3(5):1-5.

6. Mishra P, Sharma A, Mishra S. Hemisection: A conservative approach of tooth preservation. J Curr Res Sci Med. 2016;2(1):46-8.

7. Saraf AA, Patil AC. Hemisection. World J Dent. 2014:4(3):180-7.

8. Parmar G, Vashi P. Hemisection : A case-report and review. Endodontology. 2003;15:26-9.

9. American Association of Endodontist. Glossary of Endodontic Terms, Ninth Edition. 2016;9:1-50.

10. Savita S, Rajan RSS, Ambica W, Kripal K, Prakash P, Chungkhum S. Hemisection- A Baton for Extracting Teeth. Br Biomed Bull. 2015;3(4):471-5.

11. Abu-hussein M, Watted N, Abdulgani A. Hemisection A Conservative Approach For Furcation-Involved Mandibular Molar. Int J Dent Heal Sci. 2014;02(03):1012-20.

12. Behl AB. Hemisection of a Multirooted Tooth-A Case Report. 2012;1(6):6-8.

13. Chopra P, Gupta A, Tiwari R. Salvaging of Hopeless Tooth by an Interdisciplinary Approach. J Orofac Res. 2015;5(4):155-9.

14. Saad MN, Moreno J, Crawford C. Hemisection as an Alternative Treatment for Decayed Multirooted Abutment: A Case Report. J Can Dent Assoc (Tor) [Internet]. 2009;75(5):387-90. Available from: http://www.iosrjournals. org/iosr-jdms/pages/v7i4.html

15. Shah S, Modi B, Desai K, Duseja S. Hemisection - A conservative approach for a periodontally compromised tooth - A Case Report. J Adv Oral Res. 2012;3(2):31-5. 